\title{
Neutron Spin Structure Measurements in JLab Hall A
}

\author{
Jian-ping Chen, for the JLab Hall A and E94-010 Collaborations \\ Jefferson Lab, Newport News, Virginia 23606, USA
}

\begin{abstract}
Recent progress from Jefferson Lab has significantly improved our understanding of the nucleon spin structure in the high- $x$ region. Results from two experiments in Hall A are presented. The first experiment is a precision measurement of the neutron spin asymmetry, $A_{1}^{n}$, in the high$x$ (valence quark) region. The results show for the first time that $A_{1}^{n}$ becomes positive at large $x$, strongly breaking SU(6) (spin-flavor) symmetry. The data trend is in good agreement with SU(6)breaking valence quark models and with the fits to the previous world data. Combining the $A_{1}^{n}$ results with the world $A_{1}^{p}$ data, the up and down quark spins distributions in the nucleon were extracted. The results showed that for the proton the valence down quark spin is in the opposite direction from that of the proton, in disagreement with predictions of leading-order perturbative QCD models, which neglect quark orbital angular momentum.

In the second experiment, the $Q^{2}$ dependence of the moments and sum rules of the spin structure in the low to intermediate $Q^{2}$ region were measured, providing a unique bridge linking the quarkgluon picture of the nucleon and the coherent hadronic picture.
\end{abstract}

\section{INTRODUCTION}

Since the 'spin crisis' [1], substantial efforts, both theoretical and experimental, have been devoted to understanding the nucleon's spin puzzle. A new generation of experiments were carried out in the 1990s at SLAC, CERN and DESY. These experiments concluded that the quarks carry about $20-30 \%$ of the nucleon spin. The rest of the nucleon spin should come from the quark orbital angular momentum (OAM) and the gluon total angular momentum. There is almost no direct experimental information available on the quark OAM and the gluon total angular momentum. The Bjorken sum rule[2], a fundamental sum rule of the spin structure function based on QCD, was verified to an accuracy of better than $10 \%$. Attempts have been made to extract the parton spin distributions from global analyses of the polarized deep-inelastic-scattering data. The uncertainties are much larger than those of the unpolarized parton distribution due to the fact that the polarized data coverage is much less extensive than that of the unpolarized data.

Recently, the high polarized luminosity available at Jefferson Lab (JLab) has allowed the study of the nucleon spin structure at an unprecedented precision, enabling us to access the hard-to-reach valence quark (high-x) region and also to accurately map the intermediate to low $Q^{2}$ region.

The high- $x$ region is of special interest, because this is where the valence quark contributions are expected to dominate. With sea quarks and explicit gluon contributions expected not to be important, it is a clean region to test our understanding of nucleon 
structure. Relativistic constituent quark models [4] should be applicable in this region and perturbative QCD [6] is also able to make predictions in the large $x(x \rightarrow 1)$ limit. JLab experiment E99-117 measured, with high precision, the spin asymmetry $A_{1}^{n}$ in the high- $x$ region and extracted polarized quark distributions.

Related to the Bjorken sum rule, at $Q^{2}=0$ there is another sum rule for spin structure, the Gerasimov-Drell-Hearn (GDH) sum rule [7]. A generalized GDH sum rule [8] connects the GDH sum rule with the Bjorken sum rule and provides a clean way to test theories with experiments over the entire $Q^{2}$ range. JLab experiments measured the generalized GDH sum in the low to intermediate $Q^{2}$ region. These results and the results on the moments of the spin structure functions provide a bridge between the quark-gluon picture at high $Q^{2}$ to the coherent hadronic picture at low $Q^{2}$. In particular, at the low end of the $Q^{2}$ range, the results were compared with Chiral Perturbation Theory calculations and provided tests of the current understanding of the chiral dynamics of QCD.

\section{INCLUSIVE POLARIZED ELECTRON-NUCLEON SCATTERING}

For inclusive polarized electron scattering off a polarized nucleon target, the cross section depends on four structure functions, $F_{1}\left(Q^{2}, x\right), F_{2}\left(Q^{2}, x\right), g_{1}\left(Q^{2}, x\right)$ and $g_{2}\left(Q^{2}, x\right)$, where $F_{1}$ and $F_{2}$ are the unpolarized structure functions and $g_{1}$ and $\mathrm{g}_{2}$ the polarized structure functions. In the quark-parton model, $F_{1}$ or $F_{2}$ gives the quark momentum distribution and $g_{1}$ gives the quark spin distribution. Another physics quantity of interest is the virtual photon-nucleon asymmetry $A_{1}$

$$
A_{1}=\frac{g_{1}-\left(Q^{2} / v^{2}\right) g_{2}}{F_{1}} \approx \frac{g_{1}}{F_{1}} .
$$

\section{SPIN ASYMMETRIES IN THE HIGH- $X$ REGION}

To a first approximation, the constituent quarks in the nucleon are described by the SU(6) wavefunctions. The SU(6) symmetry leads to the following predictions:

$$
A_{1}^{p}=5 / 9 ; \quad A_{1}^{n}=0 ; \Delta u / u=2 / 3 ; \Delta d / d=-1 / 3 ; \quad \text { and } F_{2}^{n} / F_{2}^{p}=2 / 3 .
$$

These predictions are not expected to work in the low- $x$ region because the sea quarks and gluon contributions are not included. Experimental data on $F_{2}^{n} / F_{2}^{p}$ agree poorly with the $\mathrm{SU}(6)$ quark model predictions even in the high- $x$ region $(x>0.4)$, which is a sign that $\mathrm{SU}(6)$ symmetry is broken for valence quarks. However, a recent analysis [3] revealed a possible large uncertainty associated with the nuclear corrections to the $F_{2}^{n} / F_{2}^{p}$ data in the high- $x$ region, which makes the observed SU(6) breaking in $F_{2}^{n} / F_{2}^{p}$ less significant.

Relativistic Constituent Quark Models (RCQM) with broken SU(6) symmetry, e.g., the hyperfine interaction model [4], lead to a dominance of a 'diquark' configuration [5] with the diquark spin $S=0$ at high $x$. This implies that as $x \rightarrow 1$ :

$$
A_{1}^{p} \rightarrow 1 ; A_{1}^{n} \rightarrow 1 ; \Delta u / u \rightarrow 1 ; \text { and } \Delta d / d \rightarrow-1 / 3 .
$$


In the RCQM, the relativistic effect takes into account the quark orbital angular momentum and reduces the valence quark contributions to the nucleon spin from 1 to about $0.7-0.75$.

Another approach is with leading-order pQCD [6], which assumes the quark orbital angular momentum is negligible and leads to hadron helicity conservation. It yields the same limiting values for $A_{1}^{p}$ and $A_{1}^{n}$ as previously, but different limiting values for $\Delta u / u$ and $\Delta d / d$ :

$$
\Delta u / u \rightarrow 1 ; \text { and } \Delta d / d \rightarrow 1 .
$$

Not only are the limiting values at $x \rightarrow 1$ important, but also the behavior in the high- $x$ region. How $A_{1}^{n}$ and $A_{1}^{p}$ approach their limiting values when $x$ approaches 1 is sensitive to the dynamics of the valence quarks.

In 2001, JLab experiment E99-117 [9] was carried out in Hall A to measure $A_{1}^{n}$ with high precision in the $x$ region from 0.33 to $0.61\left(Q^{2}\right.$ from 2.7 to $\left.4.8 \mathrm{GeV}^{2}\right)$. Asymmetries from inclusive scattering of a highly polarized $5.7 \mathrm{GeV}$ electron beam on a high pressure (> $10 \mathrm{~atm}$ ) (both longitudinal and transversely) polarized ${ }^{3} \mathrm{He}$ target were measured. Beam polarization was measured with a Møller polarimeter and a Compton polarimeter. The average beam polarization was $78 \% \times(1 \pm 0.03)$. The ${ }^{3} \mathrm{He}$ target was polarized by spin exchange with optically pumped Rubidium. The average in-beam polarization was $40 \% \times(1 \pm 0.04)$. The scattered electrons were detected with two high-precision spectrometers with their standard detector packages (scintillators for trigger, vertical drift chambers for tracking, gas Cherenkov counters and shower counters for particle identification).

Parallel and perpendicular asymmetries were extracted for ${ }^{3} \mathrm{He}$. After taking into account the beam and target polarization and the dilution factor, they were combined to form $A_{1}^{3} \mathrm{He}$. Using the most recent model [10], nuclear corrections were applied to extract $A_{1}^{n}$. Final results on $A_{1}^{n}$ are shown in the left panel of Fig. 1. For clarity, not all theoretical predictions are shown. A more complete list is given in Ref. [9]

The experiment greatly improved the precision of data in the high- $x$ region. This is the first evidence that $A_{1}^{n}$ becomes positive at large $x$, showing clear SU(6) symmetry breaking. The results are in good agreement with the LSS 2001 pQCD fit to previous world data [11] (solid curve) and the statistical model [12] (long-dashed curve). The trend of the data is consistent with the RCQM predictions (the shaded band). The data disagree with the predictions from the leading-order pQCD models (short-dashed and dash-dotted curves).

Assuming the strange sea quark contributions are negligible in the region $x>0.3$, the polarized quark distribution functions $\Delta u / u$ and $\Delta d / d$ were extracted from our neutron data combined with the world proton data. The results are shown in the right panel of Fig. 1, along with predictions from the RCQM (dot-dashed curves), leading-order pQCD (short-dashed curves), the LSS 2001 fits (solid curves) and the statistical model (longdashed curves). The results agree well with RCQM predictions as well as the LSS 2001 fits and statistical models but are in significant disagreement with the predictions from the leading-order pQCD models assuming hadron helicity conservation. This suggests that effects beyond leading-order pQCD, such as the quark orbital angular momentum, may play an important role in this kinematic region. 

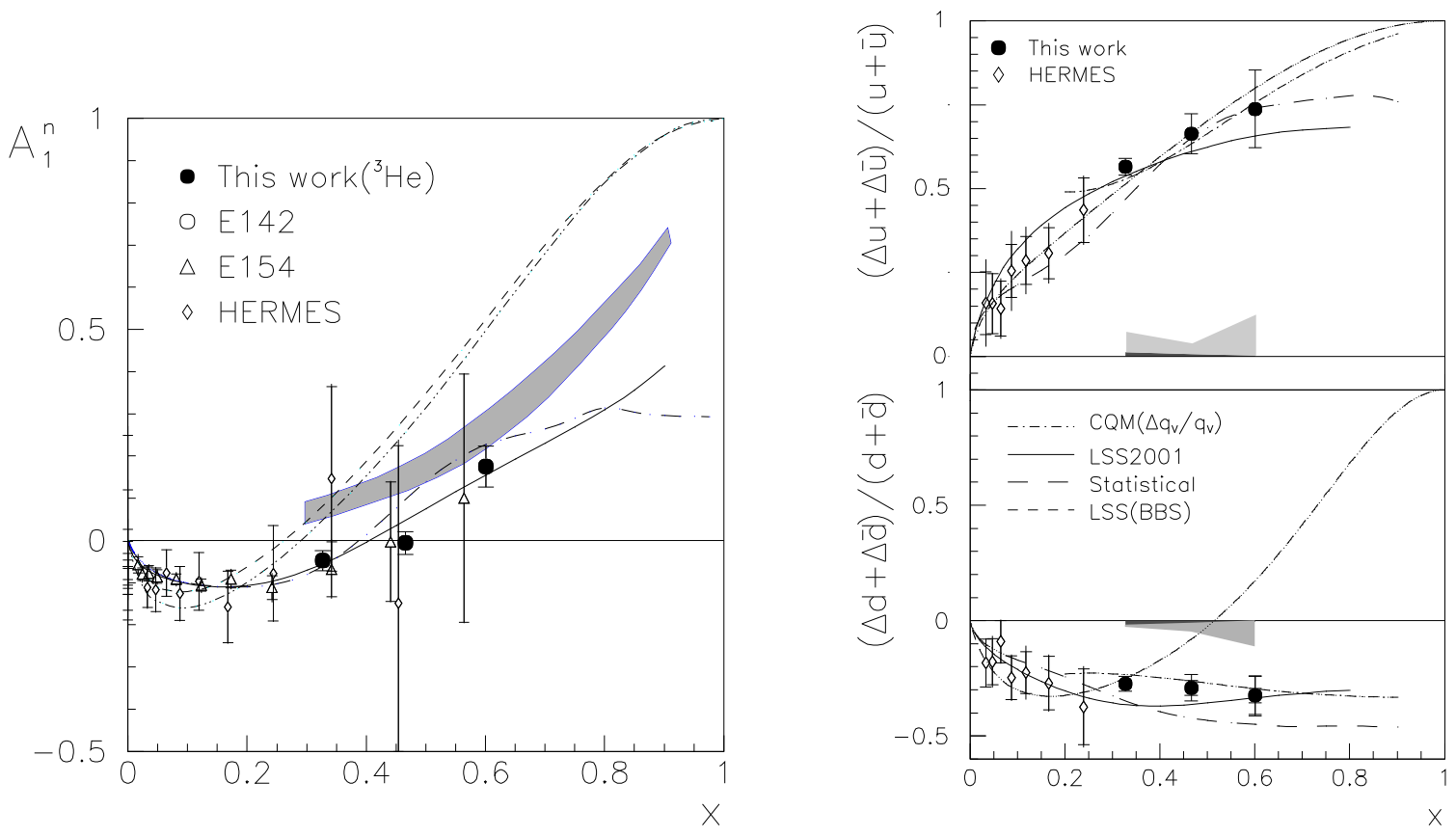

Fig. 1: $\mathrm{A}_{1}^{n}, \Delta u / u$ and $\Delta d / d$ results compared with the world data and theoretical predictions.

\section{GENERALIZED GDH SUM AND MOMENTS OF THE SPIN STRUCTURE FUNCTIONS}

JLab E94-010 [13] measured the generalized GDH sum and the moments of the neutron spin structure functions $\Gamma_{1}$ and $\Gamma_{2}$ in the low to intermediate $Q^{2}$ range using a polarized electron beam on a polarized ${ }^{3} \mathrm{He}$ target. The measurement of doubly-polarized inclusive cross sections was performed at five beam energies from 0.86 to $5.1 \mathrm{GeV}$ at a scattering angle of $15.5^{\circ}$. Parallel and perpendicular cross-section differences were obtained, from which $g_{1}, g_{2}$ and $\sigma_{T T}, \sigma_{L T}$ for ${ }^{3} \mathrm{He}$ were extracted. Interpolation to constant $Q^{2}$ values was performed and the GDH integrals were formed from pion threshold to $W^{2}=4$ $\mathrm{GeV}^{2}$. Finally, nuclear corrections [14] were applied, to extract the GDH integrals for the neutron. The results are shown in the left-top panel of Fig. 2. The higher energy contributions (for $W^{2}$ from 4 to $1000 \mathrm{GeV}^{2}$ ) were estimated using the parameterization of Thomas and Bianchi [15].

These data show a smooth but dramatic change in the value of the generalized GDH sum from what was observed at high $Q^{2}$. While not unexpected from phenomenological models, these data illustrate the sensitivity to the transition from partonic to hadronic behavior. The measured values of the first moment of $g_{1}^{n}$ are shown in the left-middle panel of Fig. 2, along with the world data from SLAC and HERMES. Also shown are Chiral Perturbation Theory calculations and several model predictions. These data provide a precision data base for twist expansion analysis at the higher end of the $Q^{2}$ range, a check for Chiral Perturbation Theory (ChPT) calculations [16] at the low end 
of the $Q^{2}$ range, and establish an important benchmark against which one can compare future calculations (such as Lattice Gauge Theory calculations). The measured values of the first moment of $g_{2}^{n}$ are shown in left-bottom panel of Fig. 2. These results indicate the first validation of the Burkhardt-Cottingham sum rule [17], $\Gamma_{2}=0$.
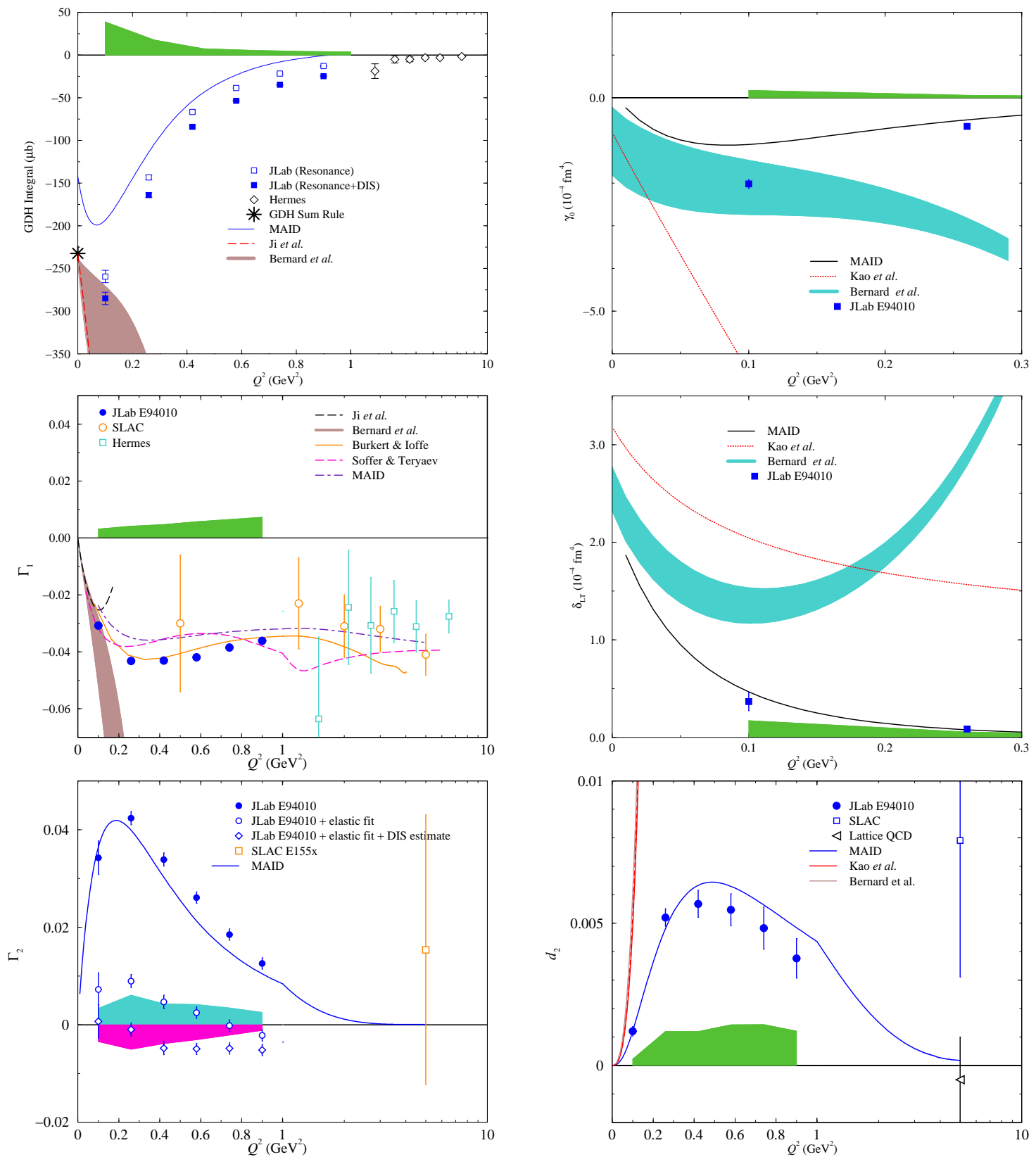

Fig. 2: Comparisons of E94-010 results with world data, ChPT calculations and model calculations.

Higher $\left(x^{2}\right.$ weighted) moments of the spin structure functions are related to generalized forward spin polarizabilities $\gamma_{0}, \delta_{L T}$ and the color polarizability $d_{2}$ [13]. The right panels of Fig. 2 show the E94-010 results on $\gamma_{0}, \delta_{L T}$ and $d_{2}$, and the ChPT calculations at low $Q^{2}$, MAID model [18] predictions at low to intermediate $Q^{2}$ and the Lattice QCD prediction at high $Q^{2}$. The relativistic baryon ChPT with resonance shows good 
agreement with the data for $\gamma_{0}$ at $Q^{2}=0.1 \mathrm{GeV}^{2}$. However, ChPT calculations deviate significantly from the data for $\delta_{L T}$, which was expected to be an excellent candidate to check Chiral dynamics of QCD since it was not sensitive to the dominating resonance $(\Delta)$ contributions. This disagreement presents a real challenge to theorists.

A new experiment [19] will extend the generalized GDH sum measurements to very low $Q^{2}$ (down to $Q^{2}=0.02 \mathrm{GeV}^{2}$ ), below the turn-around point predicted by calculations (at $Q^{2} \approx 0.1 \mathrm{GeV}^{2}$ ). ChPT calculations will be extensively tested at low $Q^{2}$ where they are expected to be applicable. Extrapolation to the real photon point provides an alternative way to test the original GDH sum rule. Data taking was completed in the summer of 2003. Analysis is underway.

In summary, with high polarized luminosity, JLab has provided a set of high-precision data to study the nucleon spin structure in a wide kinematic range, which sheds light on the valence quark structure and helped to understand the transition region between perturbative and non-perturbative regions of QCD.

\section{ACKNOWLEDGMENTS}

The work presented was supported in part by the U. S. Department of Energy (DOE) contract DE-AC05-84ER40150 Modification NO. M175, under which the Southeastern Universities Research Association operates the Thomas Jefferson National Accelerator Facility.

\section{REFERENCES}

1. B. W. Filippone and X. Ji, Adv. Nucl. Phys. 26, 1 (2001)

2. J. D. Bjorken, Phys. Rev. 148, 1467 (1966); Phys. Rev. D1, 465 (1970)

3. W. Melnitchouk and A. W. Thomas, Phys. Lett. B 377, 11 (1996); G. Petratos, these proceedings

4. N. Isgur, Phys. Rev. D59, 034013 (1999)

5. B. -Q. Ma, Phys. Lett. B 375, 320 (1996)

6. S. Brodsky, M Burkhardt and I. Schmidt, Nucl. Phys. B441, 197 (1995)

7. S. B. Gerasimov, Yad. Fiz. 2, 839 (1965).

S. D. Drell and A. C. Hearn, Phys. Rev. Lett. 162, 1520 (1966)

8. X. Ji and J. Osborne, J. Phys. G27, 127 (2001)

9. X. Zheng, et al., Phys. Rev. Lett. 92, 012004 (2004); X. Zheng, et al., nucl-ex/0405006 to be published in Phys. Rev. C

10. F. Bissey, et al., Phys. Rev. C 65, 064317 (2002)

11. E. Leader, A. V. Sidorov and D. B. Stamenov, Eur. Phys. J. C 23, 479 (2002)

12. C. Bourrely, J. Soffer and F. Buccella, Eur. Phys. J. C 23, 487 (2002)

13. M. Amarian, et al., Phys. Rev. Lett. 89, 242301 (2002); M. Amarian, et al., Phys. Rev. Lett. 92, 022301 (2004); M. Amarian, et al., nucl-ex/0406005 to be published in Phys. Rev. Lett.

14. C. Ciofi degli Atti and S. Scopetta, Nucl. Phys. B404, 223 (1997)

15. E. Thomas and N. Bianchi, Nucl. Phys. B82 (Proc. Suppl.), 256 (2000)

16. X. Ji, C. Kao, and J. Osborne, Phys. Lett. B 472, 1 (2000); C. W. Kao, T. Spitzenberg and M. Vanderhaeghen, Phys. Rev. D 67, 016001 (2003); V. Bernard, T. Hemmert and Ulf-G. Meissner, Phys. Rev. D 67, 076008

17. H. Burkhardt and W. N. Cottingham, Ann. Phys. 56, 453 (1970)

18. D. Drechsel, S. Kamalov and L. Tiator, Phys. Rev. D 63, 114010 (2001)

19. JLab E97-110, J. P. Chen, A. Deur and F. Garibaldi, et al. 